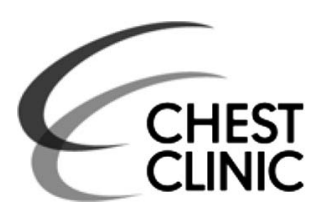

\author{
Jenny King, Malcolm Richardson, Anne-Marie Quinn, Jayne Holme, Nazia Chaudhuri
}

UHSM, Wythenshawe, UK

Correspondence to

Dr Jenny King, UHSM, Southmoor Road, Wythenshawe M239LT, UK; j.king@doctors. org.uk

Received 9 April 2016 Revised 9 June 2016 Accepted 27 June 2016 Published Online First

\title{
Bagpipe lung; a new type of interstitial lung disease?
}

\section{BACKGROUND}

Hypersensitivity pneumonitis (HP) is an inflammatory lung disease mediated by an immunological response to an inhaled antigen and can progress to disabling or fatal lung disease. It is related to occupational or other environmental exposures. In a significant proportion of patients, the antigen is often difficult to identify from the clinical history. ${ }^{1}$ This case highlights the importance of a careful clinical history including hobbies, because in this case, playing the bagpipes, we feel, was very relevant to the development of HP. We were able to isolate various fungal species from the bagpipes. There have been isolated case reports of musicians developing $\mathrm{HP}^{2}{ }^{3}$ Clinicians need to be aware of this potential trigger for developing HP, and wind instrument players need to be aware of the importance of regularly cleaning their instruments to minimise this risk.

\section{CASE PRESENTATION}

A 61-year-old man was referred to the interstitial lung disease (ILD) clinic in April 2014 with a 7-year history of dry cough and progressive breathlessness despite immunosuppressive therapy, leading to a reduction in exercise tolerance from over 10 kilometres to 20 metres. He had a prior diagnosis since 2009 of HP based on high-resolution CT (HRCT) and biopsy findings from his referring hospital. $\mathrm{He}$ did not have any exposure to birds or pigeons. His house showed no evidence of mould or water damage, and he had no symptoms of connective tissue disease. The precipitating trigger for his HP was unknown. His symptoms were insidious and progressive, with the exception of a 3-month period in 2011 when he went to live in Australia. He reported that during this time his symptoms rapidly improved and that he was able to walk 10 kilometres on the beach without stopping. On returning to the UK, his breathing deteriorated rapidly. There was no significant medical history. He was a lifelong non-smoker. As a hobby, he played the bagpipes daily. He did not take his bagpipes to Australia. $\mathrm{He}$ had been treated with prednisolone $0.5 \mathrm{mg} / \mathrm{kg}$, which had been intermittently tapered to maintain symptomatic stability. Prior to clinic review, the case was reviewed in an ILD multidisciplinary team meeting (MDT). Serial HRCT images demonstrated progressive ground-glass change and mosaic pattern. Lung function demonstrated a severe restrictive deficit with FVC of 1.48 (34\%), a 38\% decline over a 5 -year period and diffusing capacity for carbon monoxide (DLCO) of 3.21 (33\%). This had deteriorated progressively since diagnosis in 2009 when the FVC was $2.39(56 \%)$ and DLCO was 4.89 (50\%). Despite his severe restriction in lung function, he continued to play the bagpipes daily and did not require home oxygen therapy. Lung biopsy in 2009 showed upper lobe interstitial fibrosis and poorly formed granulomas. An MDT diagnosis of HP with unknown trigger was made based upon clinical history, HRCT scan and histological findings. Following specialist ILD assessment of progressive symptoms, radiological findings and of worsening restrictive lung deficit, he was commenced on $2 \mathrm{mg} / \mathrm{kg}$ azathioprine combined with maintenance prednisolone. He continued to play the bagpipes daily.

In September 2014, he was admitted with deterioration in symptoms including worsening shortness of breath for 6 weeks and low oxygen saturations when checked on his own pulse oximeter in the absence of any infective symptoms. On examination, he was afebrile, hypoxic and tachypnoeic, with bilateral fine inspiratory crepitations auscultated throughout both lung fields. Inflammatory markers were modestly elevated with a white cell count of 11.7, neutrophils of 10.85 and a $C$ reactive protein of 37 . Chest X-ray showed diffuse bilateral interstitial changes and an indistinct left heart border. The differential diagnosis was of pneumonia, PE, acute exacerbation or progression of the HP. CT pulmonary angiogram which showed no evidence of PE however demonstrated progression of fibrosis and diffuse ground-glass change. Sputum microscopy, culture and sensitivities showed normal upper respiratory tract flora and scanty Candida albicans. Sputum pneumocystis PCR and alcohol and acid-fast bacilli were negative. He was not fit enough for bronchoscopy. On admission to hospital intravenous amoxicillin/clavulanic acid, clarithromycin and co-trimoxazole were commenced to cover for atypical pneumonia and Pneumocystis jiroveci pneumonia, respectively. Posaconazole therapy was added in due to the suspicion of an underlying fungal pneumonia. During this admission, samples were taken from his bagpipes which grew a number of fungi including Paecilomyces variotti, Fusarium oxysporum, Penicillium species, Rhodotorula mucilaginosa, Trichosporon mucoides, pink yeast and Exophiala dermatitidis, identified either phenotypically or by DNA sequence analysis (figure 1). He deteriorated and died on 10 October 2014. Postmortem examination revealed diffuse alveolar damage consistent with acute respiratory distress syndrome and interstitial fibrosis, which was attributed to the history of chronic HP. Cause of death was an acute exacerbation of ILD, and no moulds or fungi were isolated at postmortem. 
Figure 1 Images of bagpipes and mycology results.

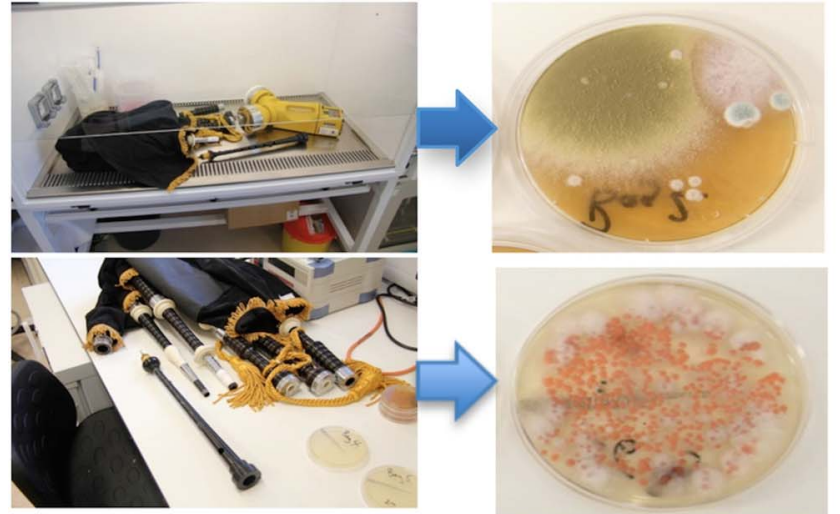

Bag inflated then squeezed while air sampling inside safety cabinet : Rhodotorula mucilaginosa

Penicillium species

Fusarium oxysporum

Stock/neck:

Mixture of Rhodotorula

mucilaginosa and

Trichosporon mucoides
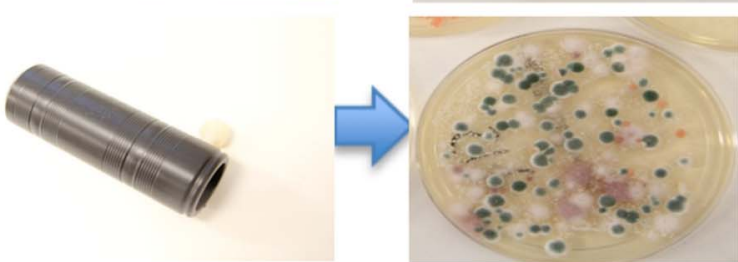

Chanter reed protector:

Penicillium species

Fusarium oxysporum

Rhodotorula mucilaginosa

\section{DISCUSSION}

This is the first case report identifying fungal exposure, from a bagpipe player, as a potential trigger for the development of HP. The clinical history of daily bagpipe-playing coupled with marked symptomatic improvement when this exposure was removed and the identification of multiple potential precipitating antigens isolated from the bagpipes make this the likely cause.

Many of the isolated fungi in this case have previously been implicated in the development of $\mathrm{HP}^{4}$ The moist environment of bagpipes promotes yeast and mould contamination, thereby making the chronic inhalation of offending antigens a likely trigger. This report highlights the importance of careful clinical history when assessing patients with respiratory symptoms. We often associate exposures to birds and pigeons, or living in environments contaminated with mould, as potential triggers for HP. In a significant proportion of patients, a trigger is not identified. In this case, playing the bagpipes, an important hobby in the history, was not initially realised as a potential trigger in the development of the disease, and subsequently, no serum-precipitating antibodies to moulds or fungi were performed. The temporal relationship with an improvement in symptoms while abroad and the subsequent identification that the patient was not playing the bagpipes highlighted this source as a potential trigger.

There have been previous case reports of HP in saxophone and trombone players attributable to isolated fungi and Candida. $^{2}{ }^{3}$ In one case described by Metzger et al, ${ }^{2}$ Ulocladium botrytis and Phoma sp were isolated from the saxophone, and subsequent serum-specific antibodies to these fungi were tested and found to be positive. The patient began regular drying of the saxophone after use, and cleaning it with disinfectant. $^{2}$ They were treated with $32 \mathrm{mg} /$ day of methylprednisolone for 1 month $^{2}$ These measures led to marked improvement of symptoms and radiology after 2 months. ${ }^{2}$ Repeat samples from the saxophone were negative for moulds or fungi. ${ }^{2}$ In a further case described by Metersky et $a l^{3}$ a trombone player developed suspected HP based on clinical and radiological findings. Symptoms showed the same temporal relationship as described in this case, as they significantly improved on cessation of playing the trombone for 2 weeks. ${ }^{2} 3$ The gentleman began to regularly clean his trombone with 91\% isopropyl alcohol, and his symptoms of cough and breathlessness resolved. ${ }^{3}$ Unfortunately, because of the fatal nature of the acute exacerbation in this case, preventative measures to ensure a period of abstinence and regular cleaning of the bagpipes were not employed. These isolated case reports highlight that wind instruments of any type could be contaminated with yeasts and moulds that act as a potential trigger for HP and highlight the need for careful history taking in identifying potential triggers. Following identification of this risk factor, the evidence of how to manage HP in wind instrument players is sparse. Both case reports demonstrate good resolution of symptoms and radiological findings with regular instrument cleaning using different methods. $^{2}{ }^{3}$ Cessation of playing the instrument has also led to symptom resolution, but can be problematic in professional players who are dependent on their ability to play to make a living. There is a lack of guidance on the correct method to clean wind instruments. One published study by Walter $e t$ al in 1958 swabbed different instruments after a variety of cleaning techniques and demonstrated least bacterial growth after cleaning instruments with a brush and commercial detergent. ${ }^{5}$ The impact of this on fungi growth was not assessed. Cleaning instruments immediately after use and allowing to drip-dry would theoretically reduce the risk of microorganism growth. There is no available literature on the optimum drug therapy to reduce the risk of disease progressions in these cases. Metzger et $a l^{2}$ suggest that a 3 -month reducing course of high-dose steroids was beneficial.

\section{LEARNING POINTS}

This is suggestive that wind instruments of any type could be contaminated with yeasts and moulds that act as a potential trigger for HP and highlights the need for careful history taking in identifying potential triggers.

Thorough clinical history exploring occupation, environmental triggers and pastimes is very important in cases of HP.

There have been isolated case reports of musicians developing HP.

Wind instrument players need to be aware of the importance of regularly cleaning their instruments and of potential risks.

Physicians should be aware of this potential risk factor and promote wind instrument hygiene. 


\section{Chest clinic}

\section{Competing interests None declared.}

\section{Patient consent Obtained.}

Provenance and peer review Not commissioned; externally peer reviewed.

\section{REFERENCES}

1 Richerson HB, Bernstein IL, Fink JN, et al. Guidelines for the clinical evaluation of hypersensitivity pneumonitis. Report of the Subcommittee on Hypersensitivity Pneumonitis. J Allergy Clin Immunol 1989;84:839-44.
2 Metzger $F$, Haccuria $A$, Reboux $G$, et al. Hypersensitivity pneumonitis due to molds in a saxophone player. Chest 2010;138:724-6.

3 Metersky ML, Bean SB, Meyer JD, et al. Trombone player's lung: a probable new cause of hypersensitivity pneumonitis. Chest 2010;138:754-75.

4 Unoura K, Miyazaki Y, Sumi Y, et al. Identification of fungal DNA in BALF from patients with home-related hypersensitivity pneumonitis. Respir Med 2011;105:1696-703.

5 Walter WG, Chaffey D. Bacteriological and cleaning studies on the mouthpieces of musical instruments. App/ Microbiol 1959;7:126-30. 